\title{
Le rôle de l'intox dans la construction de l'ethos de Marine Le Pen pendant la campagne présidentielle en France 2017
}

\section{Marine Le Pen's manipulative discourse in the 2017 French presidential election: nature and function in the construction of her ethos}

\author{
Elżbieta Pachocińska \\ Université de Varsovie \\ e.pachocinska@uw.edu.pl
}

\begin{abstract}
This study examines the nature and function of manipulative discourses by Marine Le Pen's, the candidate of Front National in the 2017 French presidential elections. It also shows how manipulative discourse legitimates her populist ethos. We adopt the discursive and rhetorical perspective (Charaudeau, 2011, 2015 ; Taguieff, 1984), which is illustrated by a corpus of texts by fact-checking journalists (les Décodeurs, Désintox) of Le Monde and Libération newspapers, as well as a video of a presidential debate.
\end{abstract}

Keywords: manipulative discourses, Marine Le Pen, the 2017 French presidential elections, populist ethos.

\section{INTRODUCTION}

«Présidentielle 2017 : une campagne plombée par les rumeurs, les intox et les fausses informations $»^{1}$ - dénonçait la presse française d'une voix presque unanime. Cette particularité de la dernière campagne présidentielle à savoir le nombre sans précédent des messages manipulatoires a été souligné par les médias et les commen-

\footnotetext{
${ }^{1} \mathrm{http}: / /$ www.lemonde.fr/les-decodeurs/article/2017/05/05/une-campagne-plombee-par-les-rumeurset-les-fausses-informations_5122623_4355770.html
} 
tateurs politiques. Ce n'est pas un phénomène nouveau dans l'espace public, les stratégies de la manipulation politique existent, on peut le dire sans exagérer, depuis l'exercice de l'art oratoire en Grèce antique. Aujourd'hui, sa nouveauté consiste en l'utilisation des supports nouveaux que lui offre la technologie numérique contribuant ainsi à la propagation globale et plus efficace des messages trompeurs. Il $\mathrm{y}$ a déjà une importante littérature qui présente ce problème de différents points de vue : politique, social, moral, philosophique, etc. Les linguistes et les chercheurs en analyse du discours ont aussi abordé les stratégies manipulatoires, en particulier dans le discours politique ou publicitaire, en articulant la complexité du phénomène (Charaudeau, 2009, 2011 ; Breton, 1997 ; Saussure, Schulz, 2005). Citons à ce propos l'observation de P. Charaudeau (2011a, pp. 5-6) :

Le discours mensonger n'existe pas en soi. (...). Il est un acte volontaire. De plus, il faut considérer que le mensonge n'a pas la même signification ni la même portée, selon que l'interlocuteur est singulier ou pluriel ou que le locuteur parle en privé ou en public. La scène publique donne un caractère particulier au mensonge. (...). L'on pourrait même dire avec quelque cynisme que l'homme politique n'a pas à dire le vrai, mais à paraitre dire le vrai $(. .$.$) .$

On est ainsi amené à se poser la question sur l'image du politique (ethos) qu'il se construit à travers ses discours qui abondent en énonciations manipulatoires. Pendant l'élection présidentielle en 2017, à chaque moment de la campagne, les journalistes décryptaient les pratiques malhonnêtes des candidats, mais c'est Marine Le Pen, la candidate du Front National qui a lancé le plus d'intox, 25 avant le débat d'entre-deux-tours et 19 pendant le débat avec Emmanuel Macron ${ }^{2}$. Pour cette raison, notre attention s'est portée sur la nature et la fonction des discours de Marine Le Pen dénommés par les médias intox et leur rôle dans la construction de l'ethos populiste.

Nous suivons la méthodologie d'analyse du discours populiste proposée par P. Charaudeau (2011) en nous appuyant sur son concept d'ethos du populiste. Vu la spécificité de ce discours, nous nous référons aussi aux recherches de P.-A. Taguieff (1984) portant sur la rhétorique du « national-populisme » qui nous permettront de situer les intox de Marine Le Pen dans une perspective plus globale des stratégies du discours populiste.

Le concept de moment discursif introduit par S. Moirand (2004), relevant de la sémantique discursive, nous servira de critère pour classer notre corpus selon deux moments importants des élections présidentielles : les intox lancés avant le débat avec Emmanuel Macron et pendant le débat du second tour. Nous proposerons de les classer selon leur nature rhétorique et la fonction dans la construction de l'image de la présidente du Front National.

\footnotetext{
${ }^{2}$ http://www.lemonde.fr/les-decodeurs/article/2017/05/03/des-intox-du-debat-entre-emmanuel-ma cron-et-marine-le-pen-verifiees_5121846_4355770.html\#kFhIsLXuK7Qqpkrg.99
} 
Pour analyser le premier moment discursif, nous avons choisi les exemples d'intox dans les articles de fact-checkeurs publiés par le journal Le Monde dans la rubrique les Décodeurs, ainsi que des articles du journal Libération de la rubrique Désintox, parus durant la campagne présidentielle 2017. Le deuxième moment discursif concernera les intox lancés par Marine Le Pen lors du débat d'entre-deuxtours, enregistré sur la vidéo.

En premier lieu, nous présenterons la spécificité du discours populiste. Ensuite, nous passerons à l'analyse de notre corpus.

\section{LA MANIPULATION ET LA DÉMAGOGIE}

Deux concepts principaux : la manipulation et la démagogie servent aux chercheurs qui analysent la rhétorique des leaders populistes à caractériser leurs stratégies discursives. Aujourd'hui, le terme populisme "fonctionne comme synonyme approximatif d'expressions telles que 'extrême droite', 'droite extrême', 'droite radicale', 'droite autoritaire', 'droite réactionnaire', c'est-à-dire, comme une désignation négative et stigmatisante. Certains l'emploient même comme substitut lexical de 'fascisme' » - remarque P.-A. Taguieff (2016). En général, le populisme sert à désigner la politique du Front National en France. P. Charaudeau (2011b, p. 103) souligne «que l'on ne peut proposer de celui-ci une définition unique, car les contextes historiques et politiques l'infléchissent de différentes façons » ses manifestations.

Les leaders populistes sont vus comme démagogues dont l'art oratoire, selon P.-A. Taguieff $(1984$, p. 114), «se réduit, dans sa fin dernière à la manipulation : pour faire agir, il faut appliquer des règles rhétoriques sans souci de vérité ou de vérifiabilité du propos ». Le démagogue fait croire qu'il représente la voix du peuple, qu'il ne se fait que le porte-parole de vox populi. Cette stratégie lui permet de légitimer sa parole, «il exploite les opinions, les croyances et les passions en cours en les renforçant, les canalysant et les orientant» (ibidem, p. 115). En suivant toujours P.-A. Taguieff (1984), le démagogue met en jeu des stratégies rhétoriques de persuasion qui sont spécifiques. Parmi les plus importantes, le chercheur cite la stratégie de la réduction des incertitudes, qui lui sert à présenter les solutions simplistes pour résoudre des problèmes sociaux et politiques. Le démagogue reprend volontiers des lieux communs de son électorat « sélectionnés et systématiquement répétés » pour construire « une façade logique et une image acceptable » en évitant des mots tabous. Dans son discours dominent les stratégies de la rétorsion par lesquelles il attaque son adversaire en utilisant ses propres arguments. La figure de «l'ennemi absolu du peuple français, selon les opinions et les valeurs admises par l'auditoire visé » est aussi fréquemment employée. Le démagogue a recours « au style de la franchise et à la profession de courage en tant que vertus opposées au mensonge et à la lâcheté des actuels responsables politiques » (ibidem, pp. 124-133). 
Nous pouvons constater que ces règles rhétoriques relevées par P.-A. Taguieff (1984) dans les discours de Jean-Marie le Pen ${ }^{3}$ sont toujours d'actualité, elles sont aussi employées par sa fille Marine Le Pen, qui est à la tête du FN depuis l'année 2011. Malgré ses efforts de « dédiaboliser » l'image de soi et de son parti, de donner l'impression que les vieilles idées d'extrême droite appartiennent déjà au passé, on constate que ses discours se placent, comme ceux de son père, dans la lignée des discours populistes. P. Charaudeau (2011b) voit aussi le caractère spécifique du discours populiste dans les stratégies de manipulations qui jouent sur les émotions du public au détriment de la raison politique, par ex. " exacerbation de la crise, dénonciation de coupables, exaltation des valeurs et apparition d'un Sauveur» (ibidem, p.106). Le leader populiste se pose comme le Sauveur, le représentant du peuple, d'où une forte présence des sujets sur l'identité nationale. Il se construit l'ethos d'authenticité («je suis tel que vous me voyez»), complété par l'ethos de puissance «C'est à cette fin (...) que son comportement oratoire se caractérise par des 'coups de gueule', des formules chocs, le maniement de l'ironie ou les dérapages verbaux » (ibidem, pp.110-111). Les chercheurs qui ont étudié les discours de l'ancien président du Front National, Jean-Marie Le Pen (Breton, 1999 ; Hailon, 2014), remarquent une forte récurrence de certains thèmes dans ses discours. F. Hailon (2014), en se basant sur les discours du Front National pendant les campagnes précédentes de 2002, de 2007 et de 2012, observe : « Le bouc émissaire est l'immigré, l'étranger, le voyou (ethnique) des quartiers qui permet l'instauration d'un discours radical, racialisant »(Hailon, 2014, p. 140). Dans toutes ces campagnes, J.-M. Le Pen se pose en tant que candidat de l'antisystème et accuse les élites politiques «de l'ensemble des maux de la société » (ibidem, p. 147). Comme on peut le voir, les mêmes sujets sont repris par sa fille, Marine Le Pen, la candidate du Front National aux présidentielles en 2017.

Pendant la campagne présidentielle de 2017, Marine Le Pen réalise son objectif politique ainsi que la ligne idéologique de son parti, Le Front National (désormais FN), en mettant en circulation des informations fausses ou des promesses électorales inapplicables. Cette orientation politique se traduit par la thématique de ses intox : l'immigration, l'insécurité, le terrorisme, les sujets qui éveillent la peur collective. Nous allons réfléchir sur les intox typiques de Marine Le Pen (désormais MLP), comme nous l'avons déjà mentionné, en suivant deux moments discursifs au sens de S. Moirand (2004, p. 375), qui, dans notre contexte, se réfèrent aux discours manipulatoires prononcés avant le débat présidentiel et pendant le débat d'entre-deux-tours avec Emmanuel Macron. Ces deux moments discursifs nous permettront de montrer des intox de nature différente. Dans le premier moment discursif, sont étudiés les intox qui mettent en valeur les présupposés idéologiques du FN, tandis que dans le débat, les intox visant la disqualification d'Emmanuel Macron.

\footnotetext{
${ }^{3}$ Le père de l'actuelle présidente du FN, Jean-Marie Le Pen, était le président du FN entre 1972-2011.
} 


\section{L’USAGE MÉDIATIQUE DU MOT INTOX}

Le $T L F i^{4}$ et le dictionnaire du CNRTL en ligne ${ }^{5}$ donnent les mêmes informations : Intox, Intoxe, subst. fém., abrév. fam. Action psychologique. Synon. matraquage. Campagne systématique de mise en condition de l'opinion publique par la diffusion d'opinions tantôt vraies tantôt fausses et plus ou moins alarmantes.

Le sens de mensonge s'est popularisé après les émissions de télévision «Double jeu » diffusée en 1991 et 1992 sur France 2, où les invités devaient se prononcer si les reportages présentés étaient vrais ou faux ${ }^{6}$. Aujourd'hui, ce mot est entré dans le vocabulaire médiatique surtout après l'apparition en 2008 de la rubrique Désintox dans le journal Libération. En 2014, c'est la rubrique les Décodeurs qui est créée dans le journal Le Monde. La technique journalistique de ces deux journaux est la même. Dans la première partie de l'article, on cite l'information erronée (intox) qui circule dans les médias. Ensuite, on décrypte son caractère fallacieux. Comme l'observe A. Rabatel (2013, p. 36), qui a analysé les intox dénoncés dans la rubrique Désintox entre 2011-2012, bien que cette contre-enquête des journalistes se fasse d' « un positionnement éthique et citoyen », elle n'est pas privée de subjectivité. Ces rubriques créées par des journalistes professionnels, qui s'inspirant de leurs collègues américains, pratiquent le genre journalistique nouveau appelé le fact-checking, à savoir la vérification des paroles des politiques et, présentation des contextes de leur apparition ${ }^{7}$. Ils répondent ainsi aux besoins des lecteurs qui sont exposés à des discours mensongers diffusés sur l'Internet et perdent confiance dans les médias traditionnels. On parle d'un phénomène social présent un peu partout dans le monde. Il s'agit de la crise moderne de confiance dans les faits présentés par les élites politiques, les partis et les médias traditionnels qui ont perdu l'autorité pour un électorat important.

\section{LE TYPE D'INTOX LANCÉS AVANT LE DÉBAT PRÉSIDENTIEL}

Dans la période d'avant le débat présidentiel, nous pouvons observer dans les intox de Marine Le Pen la dominance de la rhétorique de la simplicité, de la rhétorique $\mathrm{du}$ flou, de la propagation des informations fausses et de la manipulation des discours des autres.

\footnotetext{
${ }^{4}$ http://atilf.atilf.fr/dendien/scripts/tlfiv5/visusel.exe?27;s=3793565895;r=2;nat=;sol=6

${ }^{5} \mathrm{http}: / / \mathrm{www} . \mathrm{cnrtl}$.fr/definition/intox

${ }^{6} \mathrm{https}$ ://fr.wiktionary.org/wiki/intox

${ }^{7} \mathrm{http}: / /$ www.lemonde.fr/les-decodeurs/article/2014/03/10/la-charte-des-decodeurs_4365106_4355 770.html
} 


\subsection{LA RHÉTORIQUE DE LA SIMPLICITÉ}

Cette stratégie est présente dans des propositions de solutions politiques et sociales et de promesses électorales. Les fact-checkeurs dénoncent que les solutions invoquées par MLP n'étaient pas applicables dans ces trois cas pour des raisons différentes :

(1) Invitée de RTL mardi 18 avril, Marine Le Pen a affirmé ceci : Avec les mesures que je veux mettre en œuvre, MM. Kouachi et Coulibaly, par exemple, n'auraient pas obtenu la nationalité française car lorsqu'ils ont eu 18 ans, ils avaient un casier judiciaire long comme un jour sans pain. Donc ils n'auraient pas obtenu la nationalité et parce qu'ils sont des délinquants, ils auraient été renvoyés de France ${ }^{8}$.

La même stratégie est employée par la candidate dans la promesse suivante :

(2) Toujours sur RTL, Marine Le Pen a déclaré : « Je compte expulser immédiatement l'intégralité des fichés $\mathrm{S}$ étrangers pour lien avec le djihadisme car il n'y a aucune raison pour que nous conservions sur le territoire des gens qui représentent un danger quelconque pour la sécurité des Français »".

Ces paroles confrontées par les journalistes des Décodeurs avec la réalité juridique en France montrent que c'est une autre promesse inapplicable à la lumière de la loi actuelle en France. Cette promesse était devenue le leitmotiv de la campagne frontiste, MLP l'a reprise aussi pendant le débat avec EM. Le fait de présenter les solutions simplistes aux problèmes très complexes permet à MLP de construire son image d'un leader compétent qui sait trouver des solutions pour sauver la France du terrorisme (ethos de Sauveur).

Toujours dans la même logique se situe un autre intox de la candidate :

(3) Dans un entretien, le 17 avril, (...) Marine Le Pen a été interrogée sur l'une de ses propositions : Vous souhaitez remettre en cause l'accès à l'enseignement scolaire gratuit pour les enfants de ressortissants étrangers. Or c'est une mesure qui, vous le savez, sera très difficile à mettre en application à cause des traités internationaux qu'a signés la France et qu'a ratifiés la France?

Voici les arguments invoqués par MLP pour défendre sa mesure :

(...) l'école ne sera pas gratuite pendant deux ans quand des étrangers viendront dans notre pays. Cela existe dans d'autres pays. Je rappelle que la gratuité c'est aussi assez exceptionnel, il y a très peu de pays qui mettent en place cette gratuité totale, que ce soit pour les soins ou que ce soit pour l'école ${ }^{10}$.

\footnotetext{
${ }^{8} \mathrm{http}: / /$ www.lemonde.fr/les-decodeurs/article/2017/04/18/immigration-et-terrorisme-marine-le-penmultiplie-les-intox_5113168_4355770.html

${ }^{9}$ Ibidem.

${ }^{10}$ Ibidem.
} 
MLP laisse donc entendre que l'accès gratuit à la scolarisation pour tous les enfants serait une forme de privilège accordé en France. En réalité, il s'agit d'un droit affirmé par toutes les conventions internationales et pas seulement européennes, à savoir la Déclaration des droits de l'homme, la Convention européenne des droits de l'homme ainsi que la Convention internationale relative aux droits de l'enfant. Ses paroles signalent d'une façon indirecte que pour réaliser cette promesse, elle va faire modifier la Constitution française, et aussi elle ne va plus respecter ces conventions. Ce que d'ailleurs dans le même interview MLP promet :

(...) dans la révision constitutionnelle que je ferai par les Français, j'imposerai l'autorité de la loi nouvelle sur les traités européens et le droit dérivé antérieur ${ }^{11}$.

La question se pose donc pourquoi la candidate à la présidence lance avec une telle assurance les promesses dont la réalisation est fortement incertaine et/ou leur éventuelle application aura des conséquences graves pour la France au sein de l'Union européenne. Citons à ce propos les remarques de P. Charaudeau (2014, para. 5): «l'homme politique peut se trouver en situation de candidature électorale à des électeurs à qui il propose un projet sans savoir s'il pourra le tenir ».

MLP a recours à des stratégies démagogiques, en proposant des solutions miracles et se montrant comme la seule candidate qui puisse les mettre en vigueur. Elle construit ainsi son ethos de Sauveur, dont parle P. Charaudeau (2011). La candidate reproduit des représentations idéologiques qui plaisent à ses électeurs, au mépris des faits, ici, du droit en vigueur en France. Ce type de promesses appuie son image de leader populiste, indirectement elle attaque le gouvernement socialiste pour son impuissance de prévenir les attaques terroristes (ex. 1,2), elle réclame la priorité des droits des enfants français non issus de l'immigration (la thématique de préférence nationale), elle se montre authentique en disant des choses politiquement incorrectes sur les droits des enfants des immigrés (l'ethos d'authenticité), ainsi assure-t-elle ses électeurs qu'elle luttera contre l'immigration après son élection (l'ethos de puissance).

\subsection{LA STRATÉGIE DU FLOU}

La stratégie du flou est caractéristique pour les discours politiques à visée manipulatoire. Selon P. Charaudeau (2014, para. 6), «la stratégie du flou qui consiste à faire des déclarations suffisamment générales, alambiquées et parfois ambiguës pour qu'il soit difficile de le prendre en défaut, de lui reprocher d'avoir menti sciemment ». Nous pouvons y classer des affirmations qui manquent des précisions nécessaires pour représenter la réalité. Voici un des exemples typiques :

\footnotetext{
${ }^{11}$ Ibidem.
} 
(4) En meeting à Paris lundi 17 avril, Marine Le Pen a déclaré que les Français ont parfois moins de droits en France que des étrangers, même clandestins ${ }^{12}$.

Les journalistes remarquent que la Présidente du FN n'a pas précisé à quels «droits » elle faisait référence et confrontent cette déclaration avec les documents juridiques sur les droits de migrants en France qui ne confirment pas la thèse posée par Marine Le Pen.

Cette indétermination référentielle implicite est de nature pragmatique au sens de R. Martin (1996, p. 131). Au cas où l'on démasquerait le mensonge, MLP peut nier les faits rapportés par les journalistes, en disant qu'elle avait pensé à d'autres droits. L'intention de rester dans le vague est appuyée par l'implicite de nature linguistique introduit par l'adverbe parfois, qui présuppose dans certains cas. Selon la théorie de l'argumentation d'O. Ducrot (1980), le connecteur même introduit l'argument plus fort qui dans cette affirmation met en valeur le mot clandestin. Ce type d'intox active des présupposés idéologiques de discours de haine, en particulier des préjugés xénophobes.

\subsection{DES FAUSSES INFORMATIONS}

Le candidat qui manie des statistiques, des chiffres fait toujours l'impression d'un politique compétent, bien que ces données soient difficilement vérifiables pour le grand public. L'équipe de Désintox commente un des interview de la candidate :

(5) Marine Le Pen : une heure sur TF1, six intox

Bobards sur l'euro, l'islamisme, l'Europe ou les suppressions de postes de fonctionnaires : la présidente du FN a livré un joli pot-pourri, hier, sur TF1.

C'est un chiffre qu'elle ressort régulièrement et que nous avons récemment désintoxiqué : Marine Le Pen a expliqué une fois de plus hier soir qu'une sortie de l'Union européenne permettrait à la France de récupérer 9 milliards d'euros. Une somme qui correspond selon elle à la « contribution nette » de la France au budget européen, soit la différence entre ce que verse la France à l'Union européenne, et ce qu'elle touche de cette dernière ${ }^{13}$.

En réalité, la France verse 6 milliards d'euros, informent les journalistes en se basant sur les données du Sénat français. L'exagération de MLP sur le coût de l'Union européenne pour la France est une stratégie manipulatoire bien calculée, elle éveille les émotions fortes de ses supporteurs qui sont contre l'UE. Cet argument est construit sur le présupposé idéologique des partisans du FN et de son leader populiste à savoir que dans l'intérêt de la France est de quitter l'UE.

\footnotetext{
${ }^{12}$ Ibidem.

${ }^{13} \mathrm{http}: / /$ www.liberation.fr/elections-presidentielle-legislatives-2017/2017/04/26/marine-le-pen-uneheure-sur-tf1-six-intox_1565441
} 
Dans le même interview, nous assistons, comme le disent dans leur commentaire les fact-checkeurs, à la trumpisation de MLP, quand elle dit « les chiffres mentent » :

(6) Marine Le Pen : Tous les Français ont constaté l'explosion des prix au moment du passage à l'euro.

Anne-Claire Coudray : Ce ne sont pas ce que les chiffres disent.

Marine Le Pen : Et bien les chiffres, Madame, mentent. Et j'appelle à témoin tous ceux qui nous regardent qui ont bien vu qu'entre le passage au franc et le passage à l'euro les prix, notamment de la consommation courante, avaient explosé et évidemment considérablement grignoté leur pouvoir d'achat ${ }^{14}$.

Les journalistes argumentent qu'une hausse des prix constante depuis plusieurs années n'est pas due à l'euro en France. Cette manœuvre rhétorique (argumentum ad populum) s'inscrit aussi dans les attaques indirectes contre l'UE. MLP «appelle à témoin » l'auditoire en se référant ainsi à un présupposé manipulatoire tout le monde le sait donc c'est vrai. Elle l'impose comme une évidence de son raisonnement.

\subsection{LA MANIPULATION DES DISCOURS DES AUTRES}

Parmi les intox employés par MLP se trouvaient des citations déformées des autorités importantes pour ses adversaires. Ce type d'intox se réfère au procédé éristique déjà décrit par $\mathrm{A}$. Schopenhauer $(1830)^{15}$ comme stratagème XXX, à savoir l'argumentum ad verecundiam :

Celui-ci consiste à faire appel à une autorité plutôt qu'à la raison, et d'utiliser une autorité appropriée aux connaissances de l'adversaire. (...) On peut aussi si nécessaire non seulement déformer les paroles de l'autorité, mais carrément la falsifier ou leur faire dire quelque chose de votre invention : souvent, l'adversaire n'a pas de livre à la main ou ne peut pas en faire usage.

MLP a largement puisé dans la tradition éristique, en faisant appel à l'héritage du grand politique socialiste, Jean Jaurès ${ }^{16}$, lors d'un meeting :

(7) J'ai retrouvé un texte du XIX ${ }^{\mathrm{e}}$ siècle qui disait : «Ce que nous ne voulons pas, c'est que le capital international aille chercher la main-d'œuvre sur les marchés où elle est le plus avilie, humiliée, dépréciée, pour la jeter sans contrôle et sans réglementation sur le marché français, et pour amener partout dans le monde les salaires au niveau des pays où ils sont le plus bas ». Vous admettrez avec moi que la gauche a bien changé. L'auteur de cette exhortation s'appelait Jean Jaurès ${ }^{17}$.

${ }^{14}$ Ibidem

${ }^{15}$ Voir : stratagème XXX.

${ }^{16}$ Jean Jaurès (1859-1914).

${ }^{17} \mathrm{http}$ ://www.liberation.fr/desintox/2017/04/28/au-menu-des-intox-fn-du-jour-jaures-la-turquie-lesaffaires_1566102 
Les journalistes du Desintox confrontent cette citation avec le discours réellement prononcé par Jean Jaurès au Parlement français en février 1894 pour montrer que MLP « lui fait ainsi dire l'inverse de sa pensée ». Cet intox est utilisé pour amener les électeurs à tirer une conclusion que « Jaurès aurait voté Front National $»^{18}$.

\section{LE DÉBAT D'ENTRE-DEUX-TOURS : DEUX VISIONS POLITIQUES QUI S'AFFRONTENT}

Le moment culminant de la campagne présidentielle, c'était le débat d'entredeux-tours à la télévision le 3 mai 2017 avec Emmanuel Macron, candidat d'En Marche. Pendant ce débat de deux heures et vingt minutes d'échange, les factcheckeurs ont compté 19 intox sur tous les sujets ${ }^{19}$. Le débat d'entre-deux-tours est un événement le plus important des élections présidentielles :

(...) ce rituel médiatico-politique de la Cinquième République est perçu, dans les représentations collectives comme une arène où s'opposent et s'affrontent deux duellistes, candidats à la présidence. (...) dans cette situation de communication bien particulière, les deux finalistes se trouvent dans une problématique identitaire complexe (Dupuy, Marchand, 2016, para. 1).

Le rôle d'image positive (ethos positif) que les candidats doivent construire d'eux-mêmes pendant le débat final pour attirer le plus d'électeurs est aussi accentué par d'autres analystes du discours politique. P. Charaudeau (2015, pp. 109-110) remarque que le débat présidentiel « est une affaire d'images : images de légitimité, de crédibilité, de personnalité (caractère, puissance, subtilité, intelligence, séduction). Ces images passent essentiellement par le langage (...)». Les images de légitimité visent la construction d'une position d'autorité institutionnelle ou personnelle dans le discours, celle-ci relève de l'identité sociale. Les images de crédibilité servent à construire une image personnelle (ethos) de sincérité, ce qui attribuerait au discours un caractère crédible. Les images de personnalité servent aux politiques à montrer sa personne à travers le discours. Généralement parlant, dans ses interventions publiques «l'orateur modèle son ethos sur les représentations collectives qui revêtent aux yeux de ses interlocuteurs une valeur positive et qui sont susceptibles de produire sur eux l'impression appropriée aux circonstances » - résume Ruth Amossy (1999, p. 133).

\footnotetext{
${ }^{18} \mathrm{http} / / /$ www.liberation.fr/elections-presidentielle-legislatives-2017/2017/04/26/marine-le-pen-uneheure-sur-tf1-six-intox_1565441

${ }^{19} \mathrm{http}: / / w w w . l e m o n d e . f r / l e s-d e c o d e u r s / a r t i c l e / 2017 / 05 / 03 /$ des-intox-du-debat-entre-emmanuel-ma cron-et-marine-le-pen-verifiees_5121846_4355770.html\#kFhIsLXuK7Qqpkrg.99
} 
On peut se demander de quelle façon la présidente du FN montre son ethos positif pendant le débat d'entre-deux-tours avec EM. Voici les commentaires médiatiques dominants après le débat :

(8) Marine Le Pen noie le débat sous une avalanche d'intox (titre $)^{20}$.

(9) [Elle] a aboli en deux heures une ligne politique qu'elle avait mis dix ans à construire $^{21}$.

(10) Deux heures trente d'invective et de désaccords : le débat de l'entre-deux-tours entre Emmanuel Macron et Marine Le Pen a été d'une brutalité inédite et souvent confus ${ }^{22}$.

Nous allons nous concentrer sur les intox qui s'inscrivent dans la stratégie rhétorique d'argumentum ad hominem ${ }^{23}$, c'est-à-dire des arguments visant le discrédit de la personne en mettant en doute par ex. ses connaissances, son intelligence, en montrant une contradiction de ses idées et ses motifs «afin de le [le politique] jeter dans l'embarras et de l'amener à réviser son discours et ses positions. Cette argumentation n'a rien d'émotionnel, à la différence de l'attaque personnelle ( $\mathrm{ad}$ personam), (...) qui s'accompagne forcément d'une décharge émotionnelle » (Plantin, 2016). Elle est considérée comme des paralogismes ou des sophismes qui ne respectent pas de règles de la discussion critique. Cette stratégie semble être choisie par MLP pour empêcher un débat de fond sur les vrais problèmes des Français. En plus, elle voulait aussi éveiller des émotions fortes de l'auditoire et surtout déstabiliser son adversaire. Référons-nous encore une fois à A. Schopenhauer (1830) et plus précisément à son stratagème VIII intitulé « Fâcher l'adversaire : Provoquez la colère de votre adversaire : la colère voile le jugement et il perdra de vue où sont ses intérêts ».

\subsection{LES INTOX COMME ARGUMENTUM AD HOMINEM}

\subsubsection{LES ACCUSATIONS NON-JUSTIFIÉES}

MLP commence le débat par des attaques contre EM, en disant qu'il est «le candidat de la mondialisation sauvage, de l'uberisation, de la précarité, de la brutalité sociale, de la guerre de tous contre tous, du saccage économique notamment de nos grands groupes, de dépècement des grands intérêts économiques, du communautarisme, tout cela piloté par M. Hollande $»^{24}$.

\footnotetext{
${ }^{20} \mathrm{http}: / / \mathrm{www}$. liberation.fr/elections-presidentielle-legislatives-2017/2017/05/04/marine-le-pen-noiele-debat-sous-une-avalanche-d-intox_1567143

${ }^{21} \mathrm{http} / / / \mathrm{www}$. lejdd.fr/politique/au-debat-marine-le-pen-a-commis-une-triple-erreur-3322091

${ }^{22} \mathrm{http}: / /$ www.sudouest.fr/2017/05/04/le-debat-macron-le-pen-decrypte-80-de-ces-sourires-sont-defacade-3416549-6121.php

${ }^{23}$ Nous n'allons pas entrer dans les débats des chercheurs qui définissent l'argumentum ad personam et ad hominem souvent d'une façon contradictoire. Pour plus de détails voir G. Gauthier, 1995.

${ }^{24} \mathrm{http}$ ://www.lci.fr/elections/replay-revivez-1-integralite-du-debat-le-pen-macron-entre-deux-tours2050855.html
} 
De manière générale, elle a repris les opinions sur EM qu'elle avançait pendant toute la campagne électorale ${ }^{25}$.

Dans le chapitre consacré à l'économie, MLP a souligné à plusieurs reprises qu'EM était le ministre de l'Économie au gouvernement de F. Hollande. Elle a recours à des accusations qui se basent sur les prémisses fausses.

(11) Des entreprises que vous avez fait acheter par les grands groupes américains au détriment de l'intérêt national ALSTOM, (...), il y en a tout une série d'autres avec le conflit d'intérêts dans la banque où vous avez travaillé. Vous n'avez pas d'esprit national, vous ne pensez pas à l'intérêt de la nation (...). Ce sont des entreprises que vous avez vendues. SFR par exemple, à votre ami M. Drahi, le patron de BFM. 6000 emplois perdus. (...)

Dans votre société, tout est à vendre et tout est à acheter. Les hommes, les ventres, sont à vendre et à acheter ${ }^{26}$.

EM proteste vivement en disant que ce sont des mensonges, qu'il n'était pas ministre quand SFR a été vendue. En plus, «SFR était la propriété d'un groupe totalement privé». Pour cette raison, il a agi en conformité avec la loi «nous sommes dans un état où la propriété privée est respectée »-a argumenté EM.

La candidate voulait présenter EM en tant que ministre de l'économie qui ne se souciait pas des salariés en vendant des usines et des entreprises nationales aux capitaux privés et étrangers, qui dans la suite licenciaient les salariés français. Les mêmes accusations sont lancées par MLP à propos des chantiers STX de SaintNazaire. EM rétorque, « Vous dites une grande bêtise. Ils n'ont pas été vendus ». En plus, il n'était pas ministre, ce qu'il a répété avec insistance pendant l'affrontement.

Il est intéressant à noter les réactions d'EM aux propos mensongers de MLP : « Je voudrais rétablir la vérité sur ce que MLP a dit [sur les taxes imposées aux ouvriers, aux retraités] », «c'est faux », «Je prends les Français et les Françaises pour les adultes. Je ne leur mens pas, vous mentez en permanence $»^{27}$. Comme on peut le voir, il essayait de déconstruire l'image de MLP en démontrant que ces attaques sont des purs mensonges.

\subsubsection{LES INTOX BASÉS SUR LES RUMEURS}

P. Charaudeau (2009, p. 11) observe que la rumeur est une manipulation involontaire dont la source énonciative est inconnue, donc non imputable. Dans le débat,

\footnotetext{
${ }^{25} \mathrm{http}: / /$ www.liberation.fr/debats/2017/04/18/macron-pourquoi-tant-de-haine_1563463

${ }^{26} \mathrm{http} / / / \mathrm{www} .1 \mathrm{ci}$. fr/elections/replay-revivez-1-integralite-du-debat-le-pen-macron-entre-deux-tours2050855.html

${ }^{27} \mathrm{http} / / /$ www.lci.fr/elections/replay-revivez-l-integralite-du-debat-le-pen-macron-entre-deux-tours2050855.html
} 
MLP a saisi l'occasion de reprendre les propos diffamatoires qui circulaient sur les sites internet sans les vérifier, ce qui montre sa mauvaise intention et la volonté de disqualifier son adversaire par tous les moyens.

(12) MLP : J'espère que l'on n'apprendra pas que vous avez un compte offshore au Bahamas...

Comme le scandale attire toujours l'attention du public, MLP a relayé cette rumeur dans le présupposé de sa soi-disant question ${ }^{28}$.

\subsubsection{LES GÉNÉRALISATIONS DIFFAMATOIRES}

Ce type d'intox se base sur le raisonnement par l'induction connu en rhétorique comme un raisonnement fallacieux, c'est-à-dire allant du particulier au général :

(13) Il faut éradiquer l'idéologie du fondamentalisme islamiste dans notre pays. Mais vous ne le ferez pas, parce que vous êtes soumis à eux, M. Macron. Parce qu'ils vous tiennent. (...) On l'a vu avec l'affaire Saou. Clairement, vous avez été mis devant le chantage, soit vous gardiez M. Saou qui était radical islamiste soit UOIF appelle à vous battre. Et vous avez préférez conserver ce soutient plutôt que de prendre le risque d'exprimer votre condamnation la plus formelle de ses dérives qui sont des dérives extrêmement lourdes ${ }^{29}$.

MLP fait ici référence à « l'affaire Saou », du nom d'un membre d'En Marche dans le Val-d'Oise qui a été suspendu de ses fonctions pour une série de post Facebook sur l'islamisme dénoncés comme ambigus. C'est une reprise des accusations déjà présentes dans la campagne qui prenaient parfois le sens d'insinuations complotistes. Comme le dépistent les médias, c'est EM qui a fait naître une polémique par un tweet positif sur cette personne qu'il devait par la suite expliquer. «La droite manipule un off de Macron pour l'accuser de sympathies 'islamistes' »-a titré un de ses articles Libération $^{30}$.

\subsubsection{LES FAUSSES CITATIONS}

Pendant le débat d'entre-deux-tours, cette fausse citation attribuée à François Hollande a servi à MLP de faire ressortir les idées soi-disant socialistes d'EM.

\footnotetext{
${ }^{28}$ Voir le commentaire de MLP le jour après le débat, https://www.youtube.com/watch?v=7psnel LHBwY

${ }^{29} \mathrm{http}: / / \mathrm{www} .1 \mathrm{ci}$. fr/elections/replay-revivez-l-integralite-du-debat-le-pen-macron-entre-deux-tours2050855.html

${ }^{30}$ Ibidem.
} 
(14) MLP : Comme vous êtes socialiste, vous allez dire que « Ça ne coûte rien, c'est l'État qui paye ».

Après le débat, la presse rappelait que cette insinuation « remonte à novembre 2014. À l'époque, des milliers d'internautes avaient reproché cette phrase à F. Hollande alors qu'il n'a jamais tenu ces propos, comme le signalait L'Express ${ }^{31}$.

Cette attaque rappelle au public qu'EM a été le ministre dans le gouvernement socialiste, donc selon la logique de l'argumentation de MLP, il partage aussi les idées socialistes. Elle passe sous silence le fait que c'est justement à cause des divergences entre les socialistes et EM que ce dernier a quitté le gouvernement socialiste.

\section{CONCLUSION}

Les intox analysés représentent les différentes facettes du mensonge politique mis au service de l'idéologie populiste. Instrumentalisés dans les stratégies rhétoriques et éristiques, ils permettent à la présidente du $\mathrm{FN}$ de se positionner envers son adversaire. Paradoxalement, les intox servent à légitimer et à renforcer sa position d'autorité, ainsi qu'à la rendre crédible aux yeux de son électorat. Pour expliquer ce phénomène, nous pouvons avancer plusieurs raisons. Tout d'abord, les intox se basent sur les vieux présupposés idéologiques du FN au sujet de l'immigration, de L’Union européenne, de l'euro, etc. qui sont communément admis par ses électeurs. Dans ce sens, MLP se légitime comme représentante du peuple. Les intox introduisant les fausses informations sont employés souvent comme des outils polémiques (« les chiffres mentent ») pour éveiller les émotions du public, en particulier dans les attaques contre le gouvernement, en l'accusant de son impuissance dans la lutte contre le terrorisme, contre la crise migratoire, etc. Ils montrent le conflit idéologique entre les adversaires.

La rhétorique de la simplification, bien qu'elle mette en scène des solutions et des promesses électorales inapplicables, est fréquemment employée par MLP, parce qu'elle exprime les revendications des électeurs du FN. Dans le débat présidentiel, les intox structurent aussi l'argument ad hominem, la stratégie de la disqualification d'EM à travers laquelle se réalise la doxa populiste plus générale qui implique le rejet des élites politiques et financières. Ils deviennent un moyen rhétorique de la confrontation idéologique permettant d'exprimer la colère du peuple.

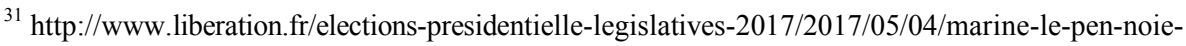
le-debat-sous-une-avalanche-d-intox_1567143
} 


\section{BIBLIOGRAPHIE}

Amossy, R. (1999). L'ethos au carrefour des disciplines : rhétorique, pragmatique, sociologie des champs. In R. Amossy (éd.), Images de soi dans le discours (pp. 127-154). Paris : Delachaux et Niestlé S.A.

Breton, P. (1997). La parole manipulée. Paris : La Découverte.

Breton, P. (1999). La "préférence manipulatoire» du président du Front national. Mots. Les langages du politique, 58, pp. 101-125. Retrieved from http://www.persee.fr/doc/mots_0243-6450_1999_num_ $58 \_1 \_2527$

Charaudeau, P. (2009). Le discours de manipulation entre persuasion et influence sociale. In Acte du colloque de Lyon. Retrieved from http://www.patrick-charaudeau.com/Le-discours-de-manipulationentre.html

Charaudeau, P. (2011a). Le discours politique ou le pouvoir du langage. Retrieved from http://www. youscribe.com/BookReader/Index/304525?documentId=277199

Charaudeau, P. (2011b). Réflexions pour l'analyse du discours populiste. Mots, 97, pp. 101-116. DOI: $10.4000 /$ mots. 20534

Charaudeau, P. (2014). L'art de mentir en politique. Sciences Humaines, 256. Retrieved from http://www.patrick- charaudeau.com/L-art-de-mentir-en-politique.html

Charaudeau, P. (2015). Le débat présidentiel. Un combat de mots. Une victoire aux points. Langage et Société, pp. 109-129. DOI: 10.3917/ls.151.0109

Ducrot, O. et al. (1980). Les mots du discours. Paris : Minuit.

Dupuy, P.-O., Marchand, P. (2016). Les débat d'entre-deux-tours de l'élection présidentielle française (1974-2012) au prisme des stratégies discursives : du monopole du cœur à la doxa économicocomptable. Mots. Les langages du politique, 112, pp. 69-79. Retrieved from http://mots.revues. org $/ 22472$

Hailon, F. (2014). L'ordre idéologique. Éléments de cognition politique. Paris : L'Harmattan.

Martin, R. (1996). Sur l'implicite dans le langage ordinaire. Le Français Moderne, LXIV, 2, pp. 129-135.

Moirand, S. (2004). La circulation interdiscursive comme lieu de construction de domaines de mémoire par les médias. In J. M. Lopez Muñoz, S. Marnette, L. Rosier (éds), Le discours rapporté dans tous ses états (pp. 373-385). Paris : L'Harmattan.

Plantin, Ch. (2016). Dictionnaire de l'argumentation. Une introduction aux études d'argumentation. Lyon : ENS.

Rabatel, A. (2013). Les apports de l'analyse des discours médiatiques : de l'interprétation des données à la critique des pratiques discursives et sociales. Retrieved from http://www.diacronia.ro/ro/indexing/ details/A1711/pdf

Saussure, L. de, Schultz, P. (2005). Manipulation and Ideologies in the Twentieth Century: Discourse, language, mind. Amsterdam-Philadelphia : John Benjamins Publishing Company.

Schopenhauer, A. (1830). L'art d'avoir toujours raison. Retrieved from https://www.les-crises.fr/wpcontent/uploads/2015/07/1-art-d-avoir-toujours-raison-d-apres-schopenhauer.pdf

Taguieff, P.-A. (1984). La rhétorique du national-populisme. Les règles élémentaires de la propagande xénophobe. Retrieved from http: //www.persee.fr/doc/mots_0243-6450_1984_num_9_1_1167

Taguieff, P.-A. (2016). Le " populisme », ce mot passe-partout. Retrieved from http://www.lefigaro.fr/vox/ politique/2016/11/24/31001-20161124ARTFIG00303-pierre-andre-taguieff-le-8220populisme8221ce-mot-passe-partout.php

\section{BASE DE DONNÉES LEXICALES}

CNRTL, http://www.cnrtl.fr/definition/intox 


\section{DICTIONNAIRE DE LANGUE EN LIGNE}

$T L F i$, http://atilf.atilf.fr/dendien/scripts/tlfiv5/visusel.exe?27;s=3793565895;r=2;nat=;sol=6; https://fr.wik tionary.org/wiki/intox

\section{SITOGRAPHIE}

Le Monde

http://www.lemonde.fr/les-decodeurs/article/2017/05/05/une-campagne-plombee-par-les-rumeurs-et-lesfausses-informations_5122623_4355770.html

http://www.lemonde.fr/les-decodeurs/article/2017/05/03/des-intox-du-debat-entre-emmanuel-macron-etmarine-le-pen-verifiees_5121846_4355770.html\#kFhIsLXuK7Qqpkrg.99

http://www.lemonde.fr/les-decodeurs/article/2014/03/10/la-charte-des-decodeurs_4365106_4355770.html $\mathrm{http}: / / \mathrm{www}$. lemonde.fr/les-decodeurs/article/2017/04/18/immigration-et-terrorisme-marine-le-pen-multiplieles-intox_5113168_4355770.html

\section{Libération}

http://www.liberation.fr/elections-presidentielle-legislatives-2017/2017/04/26/marine-le-pen-une-heuresur-tf1-six-intox_1565441

http://www.liberation.fr/desintox/2017/04/28/au-menu-des-intox-fn-du-jour-jaures-la-turquie-les-affaires 1566102

http://www.liberation.fr/elections-presidentielle-legislatives-2017/2017/05/04/marine-le-pen-noie-le-debatsous-une-avalanche-d-intox_1567143

http://www.liberation.fr/debats/2017/04/18/macron-pourquoi-tant-de-haine_1563463

\section{D'AUTRES JOURNAUX}

http://www.lejdd.fr/politique/au-debat-marine-le-pen-a-commis-une-triple-erreur-3322091

http://www.sudouest.fr/2017/05/04/le-debat-macron-le-pen-decrypte-80-de-ces-sourires-sont-de-facade3416549-6121.php

\section{VIDÉOS}

http://www.lci.fr/elections/replay-revivez-l-integralite-du-debat-le-pen-macron-entre-deux-tours-2050 855.html

https://www.youtube.com/watch?v=7psnelLHBwY 